4-1-2008

\title{
A Refined View of Vertical Mass Transport by Cumulus Convection
}

Harmen J.J. Jonker

Delft University of Technology, h.j.j.jonker@tudelft.nl

Thijs Heus

Cleveland State University, t.heus@csuohio.edu

Peter P. Sullivan

National Center for Atmospheric Research

Follow this and additional works at: https://engagedscholarship.csuohio.edu/sciphysics_facpub

Part of the Physics Commons

How does access to this work benefit you? Let us know!

Publisher's Statement

Open Access

\section{Repository Citation}

Jonker, Harmen J.J.; Heus, Thijs; and Sullivan, Peter P., "A Refined View of Vertical Mass Transport by Cumulus Convection" (2008). Physics Faculty Publications. 227.

https://engagedscholarship.csuohio.edu/sciphysics_facpub/227

This Article is brought to you for free and open access by the Physics Department at EngagedScholarship@CSU. It has been accepted for inclusion in Physics Faculty Publications by an authorized administrator of

EngagedScholarship@CSU. For more information, please contact library.es@csuohio.edu. 


\title{
A refined view of vertical mass transport by cumulus convection
}

\author{
Harm J. J. Jonker, ${ }^{1}$ Thijs Heus, ${ }^{1}$ and Peter P. Sullivan ${ }^{2}$ \\ Received 9 November 2007; revised 22 January 2008; accepted 25 February 2008; published 5 April 2008.
}

[1] The purpose of this letter is to show that the traditional view of transport by shallow cumulus clouds needs important refinement. On the basis of a straightforward geometrical analysis of Large Eddy Simulation results of shallow cumulus clouds, we conclude (1) that the upward mass transport by clouds is strongly dominated by regions close to the edge of clouds rather than by the core region of clouds and (2) that the downward mass transport is dominated by processes just outside the cloud. The latter finding contradicts the accepted view of a uniformly descending dry environment. We therefore advocate a refined view which distinguishes between the near-cloud environment and the distant environment. The near-cloud environment is characterized by coherent descending motions, whereas the distant environment is rather quiescent and plays no significant role in vertical transport. Citation: Jonker, H. J. J., T. Heus, and P. P. Sullivan (2008), A refined view of vertical mass transport by cumulus convection, Geophys. Res. Lett., 35, L07810, doi:10.1029/2007GL032606.

\section{Introduction}

[2] The elementary view of vertical transport in a shallow cumulus cloud layer employs the fundamental notion that buoyant cloudy air moves upward with appreciable speed in relatively small columnar regions, while dry environmental air moves slowly downward, uniformly spread over a relatively large area. The corresponding upward and downward mass-fluxes compensate each other such that massconservation is satisfied. This conceptual view of cumulus convection, depicted schematically in Figure 1a, forms the basis of the successful mass-flux parameterization of cumulus clouds [e.g., Arakawa and Schubert, 1974; Asai and Kashara, 1967; Betts, 1975; Tiedtke, 1989; Siebesma and Cuijpers, 1995; Bretherton et al., 2004], in which the cloud field is represented by one effective cloud with fractional area $\sigma$ and vertical velocity $w_{c}$, yielding a cloud mass flux (omitting the density $\rho$ ) of $M_{c}=\sigma w_{c}$, and in which the environment is represented by a uniform downward velocity $w_{e}$ over a fractional area $1-\sigma$, corresponding to a mass-flux of $M_{e}=(1-\sigma) w_{e}=-M_{c}$.

[3] However, aircraft observations of cumulus clouds by, for example, Jonas [1990] and Rodts et al. [2003], have revealed that clouds generally tend to be surrounded by a sheath of air which descends with a significant velocity compared to the more distant environment. Recently,

\footnotetext{
${ }^{1}$ Department of Multi-Scale Physics, Delft University, Delft, Netherlands.

${ }^{2}$ National Center for Atmospheric Research, Boulder, Colorado, USA.

Copyright 2008 by the American Geophysical Union. 0094-8276/08/2007GL032606
}

Heus and Jonker [2008] analyzed this phenomenon by employing Large Eddy Simulations (LES) of shallow cumulus and studied in more detail the mechanism behind the formation of the descending shell of air. In line with Rodts et al. [2003], they concluded that the descending shell is caused by the strong negative buoyancy that results from just evaporated cloudy air near cloud edge ('evaporative cooling'). On the basis of their three-dimensional LES data, Heus and Jonker [2008] hypothesized that the total downward mass-flux associated with the descending shell could in principle be significant, not so much because of the magnitude of the descending motion, but rather because of the relatively large area occupied by the shell, since it surrounds clouds along their entire perimeter. The view that emanates from these results is schematically depicted in Figure 1b. In this letter we test the validity of this view, by developing a methodology that exactly quantifies the mass-flux contributions as a function of the distance to the cloud-edge. If the hypothesis is valid, a significant mass-flux contribution ought to be found near cloud-edge.

\section{Methodology}

[4] The large-eddy simulation (LES) case studied here is based on the observations made during the Small Cumulus Microphysics Study (SCMS) in August 1995 over Florida [e.g., Knight and Miller, 1998]. The domain size is $L=6.4 \mathrm{~km}$ in $x, y$ and $5.12 \mathrm{~km}$ in the vertical; the resolution is $(25 \mathrm{~m})^{2} \times 20 \mathrm{~m}$. More details about the employed LES-model and the specific LES-compilation of the SCMS-case used here are given by Neggers et al. [2003]. The novelty in the present analysis, compared to previous cloud mass-flux oriented LES-studies, like Siebesma and Cuijpers [1995] and Wang and Stevens [2000], resides in the fact that we determine mass-flux contributions in space as a function of the distance to the cloud boundary. To this end we specify for each grid-point in the cloud layer its horizontal distance to the nearest cloud-edge. The calculation of the distances is repeated for each new cloud realization. After a spin-up period of three hours we analyzed the cloud fields each minute during two hours, leading to 120 cloud field realizations. For each realization we first identify all cloud boundaries by looking in the horizontal plane for pairs of grid-boxes of which one contains liquid water and the other not. Next we calculate for each grid-box the horizontal distance $r$ to the nearest cloud-edge. When a grid-point is located inside a cloud we assign a negative value for the distance to cloudedge.

[5] Once the distances have been determined, we calculate the mass-flux contribution $m(r)$ of those points with a distance to cloud-edge between $r$ and $r+\mathrm{d} r$. Since $r$ is defined negative inside the cloud and positive in the environment, 

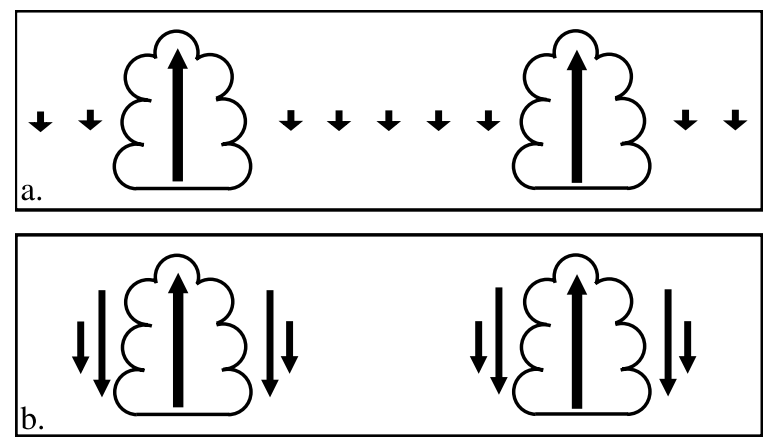

Figure 1. (a) Traditional view on cumulus convection: strong but narrow updrafts associated with cloudy air are compensated by a uniform slowly subsiding motion in the relatively broad dry environment. (b) Alternative view tested in this letter: most of the downward mass-flux takes place in the near vicinity of cloud-edges. The more distant environment plays no appreciable role in the downward mass transport.

both the cloud mass-flux $M_{c}$ and the environmental mass-flux $M_{e}$ can be retrieved from $m(r)$ by integration:

$$
M_{c}=\int_{-\infty}^{0} m(r) \mathrm{d} r \quad M_{e}=\int_{0}^{\infty} m(r) \mathrm{d} r
$$

Note that $\int_{-\infty}^{\infty} m(r) \mathrm{d} r=0$. We also determine the (fractional) area density $n(r)$, i.e. the normalized number of locations with a certain distance $r$ to cloud-edge. The fractional area of the cloudy and environmental part can be found from $n(r)$ through:

$$
\sigma=\int_{-\infty}^{0} n(r) \mathrm{d} r \quad 1-\sigma=\int_{0}^{\infty} n(r) \mathrm{d} r
$$

By definition $n(r)$ integrates to unity. The statistical reliability of $n(r)$ and $m(r)$ was improved by additionally averaging over the 120 different cloud field realizations.
[6] Conditional averages of an arbitrary (thermo)dynamic variable $\phi$ with respect to $r$ are obtained by taking the average value of $\phi$ over all grid locations having distance $r$. More formally, if $\tilde{r}(x, y)$ denotes the operator that yields the cloud-edge distance at position $(x, y)$, the conditional average of $\phi$ with respect to $r$ can be expressed as

$$
\phi(r)=\frac{L^{-2} \iint \phi(x, y, z) \delta(\tilde{r}(x, y)-r) \mathrm{d} x \mathrm{~d} y}{n(r)}
$$

where $\delta$ is the Dirac-function. In this notation $n(r)=$ $L^{-2} \iint \delta(\tilde{r}(x, y)-r) \mathrm{d} x \mathrm{~d} y$ and $m(r)=L^{-2} \iint w(x, y) \delta(\tilde{r}(x, y)-$ $r) \mathrm{d} x \mathrm{~d} y$. The average vertical velocity conditioned upon $r$ is thus $w(r)=m(r) / n(r)$. Note that, although not explicitly indicated, all quantities in equations (1-3) still depend on the height $z$.

\section{Results}

\subsection{Middle of the Cloud Layer}

[7] In Figure 2 (top) we show both the average vertical velocity $w(r)$ and the fractional area $n(r)$ as a function of $r$, the distance to the nearest cloud-edge. The results are obtained at $z=1500 \mathrm{~m}$, which is roughly the middle of the cloud layer. The graph of $w(r)$ provides a familiar picture: the deeper inside the cloud (more negative $r$ ), i.e. the closer one gets to the cloud core, the larger is the upward velocity. Near the cloud boundary $(r \rightarrow 0)$ the velocities decrease and become negative outside of the cloud $(r>0)$, nicely revealing the shell of descending air in the cloud vicinity. Further away from the cloud, one may note, the average vertical velocity goes to zero.

[8] In the same figure the fractional area $n(r)$ (solid line) has been plotted; this quantity can be viewed as the probability of being at distance $r$ to a cloud boundary. This graph is non-trivially linked to both the cloud-size distribution and the geometric distribution of clouds in space. Since $n(r)$ peaks at $r=400 \mathrm{~m}$, the graph tells us that by randomly picking a location in the cloud field at $z=1500 \mathrm{~m}$, one most
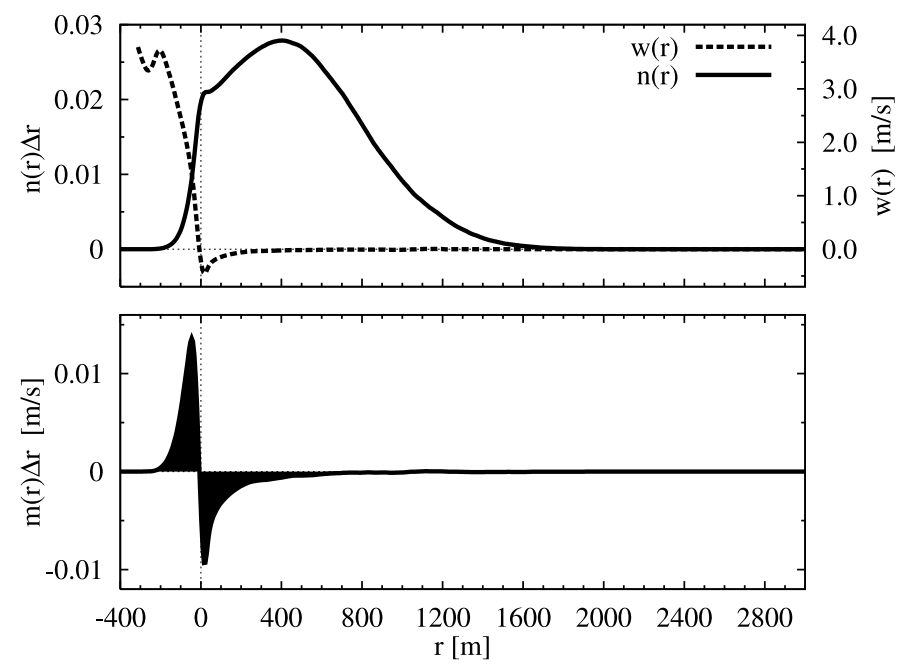

Figure 2. Results at $z=1500 \mathrm{~m}$ (middle of the cloud layer). (top) Average vertical velocity $w(r)$ (dashed line) and fractional area $n(r)$ (solid line) as a function of distance $r$ to the nearest cloud-edge. (bottom) Mass-flux contribution as a function of distance to cloud-edge. 
likely ends up in the environment at $400 \mathrm{~m}$ distance from some cloud edge. Another relevant point to note is that the number of points with large negative $r$ is quite small. This is logical for two reasons: (1) there are only few big (wide) clouds in the ensemble [e.g., Neggers et al., 2003, and references therein] and (2) true inner core points are scarce even in big clouds: also for large clouds the points near cloud-edge are a vast majority for obvious geometrical reasons. Consequently, even though the velocities in the core of big clouds are large, their effective impact on vertical mass transport is rather small.

[9] To make the latter surprising notion quantitative, we show in Figure 2 (bottom) the mass-flux contribution $m(r)$ as a function of $r$. Note that $m(r)$ can be viewed as the product of $w(r)$ and $n(r)$, i.e. the two lines in the top graph. The graph of $m(r)$ is the most important plot of this letter. There are a number of striking and unexpected aspects in this figure. First and foremost is the large negative massflux present in the near vicinity of cloud-edge $(0 \leq r \leq$ $400 \mathrm{~m}$ ). The naked eye immediately observes that the area under the curve in the region $[0,400 \mathrm{~m}]$ is of similar magnitude as the area under the curve in the region $r<0$. The latter area is equal to the cloud-mass flux $M_{c}$, as given in (1). Hence, the graph unambiguously reveals that the compensating downward mass-flux takes place in the immediate proximity of the clouds, i.e. in the collection of shells surrounding clouds, and not in the form of a weak subsiding motion uniformly distributed over the entire environmental region. The second noteworthy aspect of $m(r)$ in Figure 2 is the importance of cloud-edge for the in-cloud upward vertical mass transport, as well as the earlier mentioned subdominant role played by the cores of big clouds.

[10] In summary, it is found that the regions near cloudedge play a prominent, if not dominant, role in both the upward and downward mass transport. One of the underlying reasons is that the area occupied by cloud boundary is surprisingly large, a property related to the fractal geometry of cloud surfaces [e.g., Lovejoy, 1982; Siebesma and Jonker, 2000]. A second reason for the high contributions near $r=0$ originates from the typical power-law form of cloud-size distributions, which imply few big clouds and numerous small clouds [e.g., Neggers et al., 2003; Rodts et al., 2003].

\subsection{Height Dependence}

[11] Below we show that the behavior in the middle of the cloud layer, as discussed in the previous section, is characteristic of the entire cloud layer, with some exceptions at cloud base. To first provide some idea of the vertical structure of the cloud layer, we plot in Figure 3 the profile of the mean virtual potential temperature $\left\langle\theta_{v}(z)\right\rangle$ together with the cloud mass-flux $M_{c}(z)$ (scale on upper axis). We also calculated the cloud conditional average $\theta_{v}^{c}(z)$, i.e. $\theta_{v}$ averaged over cloudy points only. In the right column we have plotted the difference $\theta_{v}^{c}(z)-\left\langle\theta_{v}(z)\right\rangle$, which is the relevant quantity to gain insight in the buoyancy of clouds.

[12] Consistent with previous studies, one notes that the buoyancy of the clouds is rather modest $\left(\theta_{v}^{c}-\left\langle\theta_{v}\right\rangle \approx 0.1 \mathrm{~K}\right)$. At cloud base cloudy air is on average slightly negatively buoyant, indicative of the barrier that needs to be overcome before the level of free convection (LFC) is reached. At the

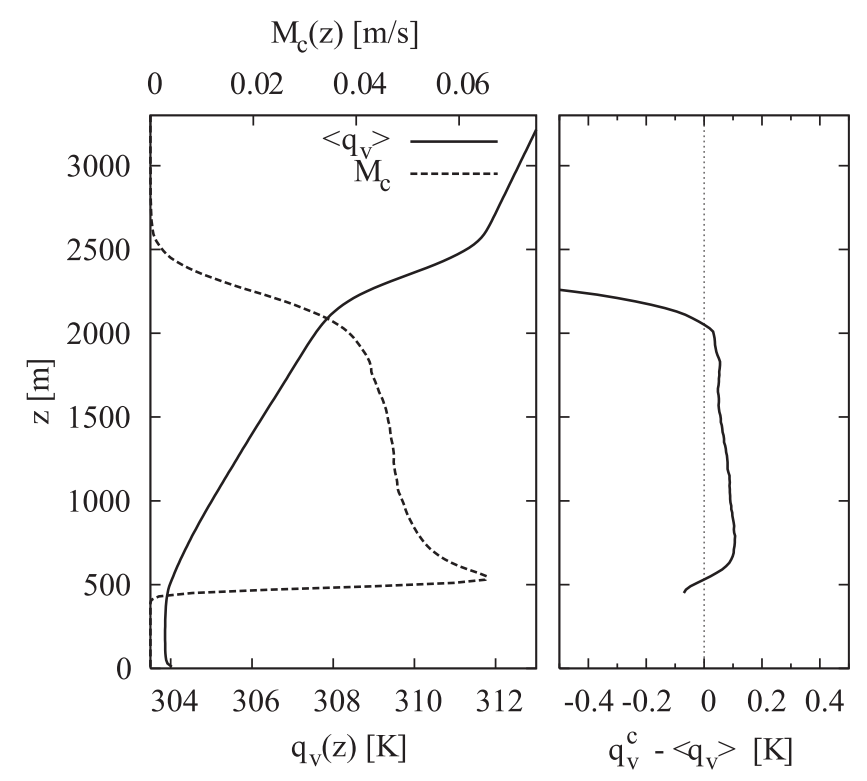

Figure 3. (left) Mean profile of the virtual potential temperature, $\left\langle\theta_{v}(z)\right\rangle$ (solid line), together with the cloud mass-flux profile $M_{c}(z)$ (dashed line). (right) Excess of cloud averaged values $\theta_{v}^{c}(z)$ with respect to the mean value $\left\langle\theta_{v}(z)\right\rangle$.

inversion $(z \approx 2300 \mathrm{~m})$ the negative buoyancy, resulting from overshooting clouds, is very clear.

[13] With the methodology introduced above it is possible to have a more detailed look at various thermodynamic variables. As an example we plot in Figure 4 (left) the average value of $\theta_{v}$ conditioned on the distance to cloud edge $r$, calculated according to (3) with $\phi=\theta_{v}$. Comparing the $\theta_{v}(r)$ values of negative $r$ with the more distant environment, one observes that cloud cores (most negative $r$ ) are most buoyant, which does not come as a surprise. For large negative $r$, the values become somewhat erratic due to the poor statistics of these points. Near cloud edge $(r=0)$ on the other hand, one can clearly observe the negative buoyancy, which is the result of cooling due to evaporation of cloudy air that has mixed with dry environmental air. This mechanism was identified as the main cause for the shell of descending air around clouds [e.g., Rodts et al., 2003; Heus and Jonker, 2008]. It is interesting to note that the near-edge in-cloud regions are consistently negatively buoyant as well. In the inversion $(z=2300 \mathrm{~m})$ the entire cloud is negatively buoyant, in line with Figure 3. In Figure 4 (middle) one can see the mass-flux contributions as a function of $r$ for various heights. On the whole, the figure provides the same message as Figure 2: that the downward mass-flux takes place in the proximity of cloud-edges and not in the form of a weak subsiding motion uniformly distributed over the environment. A small exception can be seen at cloud base, where the downward mass-flux is wider spread. But slightly higher up (from $z=700 \mathrm{~m}$ onwards, i.e. above the level of free convection), the mass-flux density profiles assume a universal shape with no significant changes until the inversion is reached. There the near-cloud environmental mass-flux becomes more negative than the upward cloud mass-flux; 

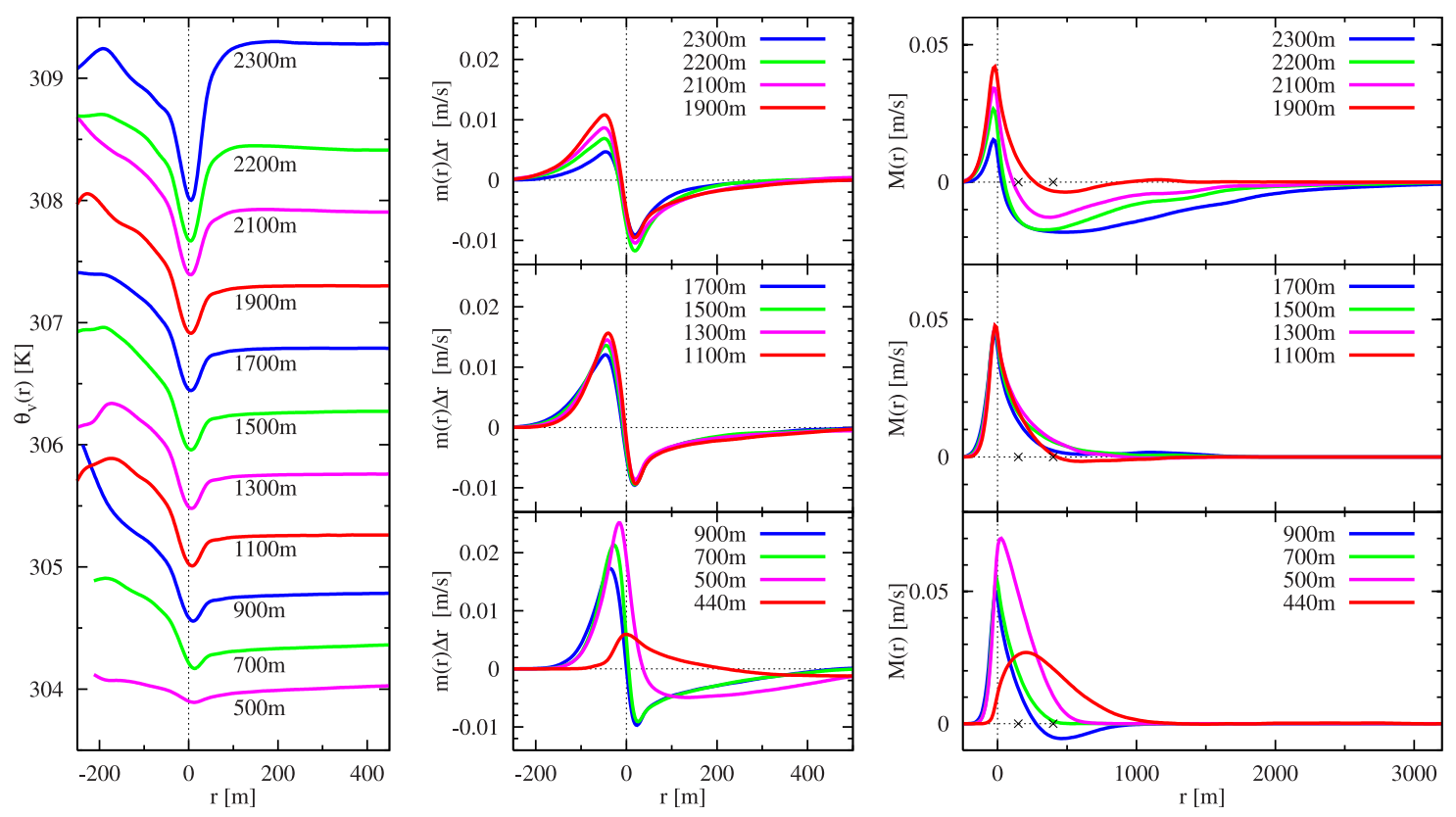

Figure 4. (left) Average value of $\theta_{v}$ as a function of the distance to cloud-edge $r$, for various heights. (middle) Mass-flux contribution $m(r)$. (right) Cumulative mass-flux $M(r)$ as defined in (4). Crosses indicate the positions $r=150 \mathrm{~m}$ and $r=400 \mathrm{~m}$, which are referred to in the text.

this can only imply that the more distant environment has a positive mass-flux, although this is hardly noticeable in the plot of $m(r)$. Yet the effect can be well observed in the right column of Figure 4, where we have plotted the the cumulative mass-flux:

$$
M(r)=\int_{-\infty}^{r} m\left(r^{\prime}\right) \mathrm{d} r^{\prime}
$$

Note that $M(0)$ is equal to the cloud mass-flux $M_{c}$ and furthermore that $M(\infty)=0$. For the inversion region one can infer from the top right graph of Figure 4 that around the clouds more mass comes back than is penetrated up by the clouds. This net downward mass transport is compensated in the distant environment where indeed a positive contribution to the mass-flux can be observed.

[14] The cumulative mass-flux plots in Figure 4 are also useful because they provide a measure as to how far one must travel from the cloud into the environment so that most of the cloud mass-flux has been compensated by the subsiding air. For example, Figure 4 (right) shows that $50 \%$ of the cloud mass-flux is already compensated for $r<150 \mathrm{~m}$ and $90 \%$ for $r$ smaller than $400 \mathrm{~m}$. This is true for the entire cloud layer, except near cloud base.

\section{Conclusions and Discussion}

[15] We have introduced a new analysis technique to examine cumulus cloud fields, in which mass-flux contributions and (thermo)dynamic variables are analyzed as a function of the horizontal distance to the nearest cloud-edge. The results of this approach show unambiguously that the cloud-edge regions play important roles in upward and downward vertical mass transport.
[16] It was demonstrated that the compensating downward mass-flux takes place in the immediate proximity of clouds, and not in the form of a weak uniformly subsiding motion. Hence it is natural to make a distinction between the near-cloud environment and the more distant environment. The latter region is found to be rather quiescent, and to have no significant contribution to the downward vertical transport. On the basis of these results we propose a refined view of vertical mass transport by cumulus convection, schematically depicted in Figure $1 \mathrm{~b}$.

[17] The refined view is relevant in at least two areas: dispersion of atmospheric compounds in a cumulus field, and cloud lateral entrainment. First, if the view of Figure 1a is correct, a tracer gas released in the distant environment may be expected to reach cloud base in a finite time since it will descend on average with velocity $w_{e}$. Figure $1 \mathrm{~b}$, on the other hand, predicts that a tracer gas released in the distant environment will linger for a very long time; the tracer will then only descend when it gets into a near-cloud environment, for example due to the formation of a new cloud near-by. These predictions can be directly tested.

[18] Secondly, with regard to lateral entrainment we stress that the air being entrained is near-cloud environmental air, the properties of which differ significantly from the properties of the distant environmental air. This notion was recently alluded to by Gerber [2006] (using the term preconditioned air) in the context of homogeneous/heterogeneous mixing in clouds. It will be insightful to apply the methodology introduced in this letter directly to aircraft measurements.

[19] Acknowledgments. This work was sponsored by the National Computing Facilities Foundation (NCF) for the use of supercomputer facilities, with financial support of NWO. The authors thank R. Verzijlbergh and F. Pols for useful discussions. 


\section{References}

Arakawa, A., and W. H. Schubert (1974), Interaction of a cumulus cloud ensemble with the large-scale environment, Part I, J. Atmos. Sci., 31, $674-701$.

Asai, T., and A. Kashara (1967), A theoretical study of compensating downward motions associated with cumulus clouds, J. Atmos. Sci., 24, $487-496$

Betts, A. K. (1975), Parametric interpretation of trade-wind cumulus budget studies, J. Atmos. Sci., 32, 2363-2382.

Bretherton, C. S., J. R. McCaa, and H. Grenier (2004), A new parameterization for shallow cumulus convection and its application to marine subtropical cloud-topped boundary layers. Part I: Description and 1D results, Mon. Weather Rev., 132, 864-882.

Gerber, H. (2006), Entrainment, mixing, and microphysics in RICO cumulus, paper presented at 12th Symposium on Cloud Physics, Am. Meteorol. Soc., Madison, Wisc.

Heus, T., and H. J. J. Jonker (2008), Subsiding shells around shallow cumulus clouds, J. Atmos. Sci., in press.

Jonas, P. R. (1990), Observations of cumulus cloud entrainment, Atmos. Res., 25, 105-127.

Knight, C. A., and L. J. Miller (1998), Early radar echoes from small, warm cumulus: Bragg and hydrometeor scattering, J. Atmos. Sci., 55, 29742992.
Lovejoy, S. (1982), Area-perimeter relation for rain and cloud areas, Science, 216, 185-187.

Neggers, R. A. J., H. J. J. Jonker, and A. P. Siebesma (2003), Size statistics of cumulus cloud populations in large-eddy simulations, J. Atmos. Sci., 60, $1060-1074$

Rodts, S. M. A., P. G. Duynkerke, and H. J. J. Jonker (2003), Size distributions and dynamical properties of shallow cumulus clouds from aircraft observations and satellite data, J. Atmos. Sci., 60, 1895-1912.

Siebesma, A. P., and J. W. M. Cuijpers (1995), Evaluation of parametric assumptions for shallow cumulus convection, J. Atmos. Sci., 52, 650666.

Siebesma, A. P., and H. J. J. Jonker (2000), Anomalous scaling of cumulus cloud boundaries, Phys. Rev. Lett., 85, 214-217.

Tiedtke, M. (1989), A comprehensive mass flux scheme for cumulus parameterization in large-scale models, Mon. Weather Rev., 177, 1779-1800.

Wang, S., and B. Stevens (2000), On top-hat representations of turbulence statistics in cloud-topped boundary layers, J. Atmos. Sci., 57, 423-441.

T. Heus and H. J. J. Jonker, Department of Multi-Scale Physics, Delft University, P.O. Box 5046, NL-2600 GA Delft, Netherlands. (h.j.j.jonker@ tudelft.nl)

P. P. Sullivan, National Center for Atmospheric Research, Box 3000, Boulder, CO 80307-3000, USA 